\title{
Evolution of wet agglomerates inside inertial shear flow of dry granular materials
}

\author{
Thanh-Trung Vo, ${ }^{1,2}$ Patrick Mutabaruka $\odot,{ }^{2}$ Saeid Nezamabadi, ${ }^{2,3}$ Jean-Yves Delenne $\odot,{ }^{3}$ and Farhang Radjai $\oplus^{2, *}$ \\ ${ }^{1}$ Bridge and Road Department, Danang Architecture University, 550000 Da Nang, Vietnam \\ ${ }^{2}$ LMGC, Université de Montpellier, CNRS, Montpellier, France \\ ${ }^{3}$ IATE, UMR1208 INRA-CIRAD-Université de Montpellier-SupAgro, 34060 Montpellier, France
}

(Received 25 November 2019; accepted 21 February 2020; published 11 March 2020)

\begin{abstract}
We use particle dynamics simulations to investigate the evolution of a wet agglomerate inside homogeneous shear flows of dry particles. The agglomerate is modeled by introducing approximate analytical expressions of capillary and viscous forces between particles in addition to frictional contacts. During shear flow, the agglomerate may elongate, break, or be eroded by loss of its capillary bonds and primary particles. By systematically varying the shear rate and surface tension of the binding liquid, we characterize the rates of these dispersion modes. All the rates increase with increasing inertial number of the flow and decreasing cohesion index of the agglomerate. We show that the data points for each mode collapse on a master curve for a dimensionless scaling parameter that combines the inertial number and the cohesion index. The erosion rate vanishes below a cutoff value of the scaling parameter. This leads to a power-law borderline between the vanishing erosion states and erosion states in the phase space defined by the inertial number and the cohesion index.
\end{abstract}

DOI: 10.1103/PhysRevE.101.032906

\section{INTRODUCTION}

Agglomerates of fine particles appear either naturally in wet soils (soil aggregates) and powders (lumps) as a result of the binding action of capillary bridges between particles [1,2], or through an agglomeration process used for the manufacture of pellets [3-6] and pharmaceutical or food products [7,8]. Once nucleated, the agglomerates interact with their surrounding granular material. The evolution of the agglomerates and their effect on the soil or powder behavior depends on the nature of these interactions [3,9-15]. There are two limit cases: (i) The agglomerate survives by keeping its shape and primary particles if its internal cohesion $\sigma_{c}$ is high due to strong cohesive bonds as compared to the forces exerted by dry granular flow around the agglomerate, and (ii) the agglomerate spontaneously disintegrates if the cohesive bonds are weak. The internal cohesion of the agglomerate is proportional to the mechanical strength measured by subjecting the agglomerates to a compression test between two platens [16-19]. The strength is basically controlled by the surface tension of the binding liquid but also by the texture of the agglomerate [20].

We consider the case in which all the surrounding particles are dry so that the agglomerate may not grow by capturing wet particles. The contact forces exerted by the dry granular flow have their origins either in the confining pressure $\sigma_{p}$ or in the particle inertia [21]. The latter can be expressed as a Bagnoldlike kinetic pressure $\sigma_{i} \sim \rho_{s}\langle d\rangle^{2} \dot{\gamma}^{2}$, where $\rho_{s}$ is the particle density, $\langle d\rangle$ is the mean particle diameter, and $\dot{\gamma}$ is the shear rate $[22,23]$. Hence, the fate of the agglomerate depends on the competing actions of $\sigma_{c}, \sigma_{i}$, and $\sigma_{p}$ or their dimensionless

*franck.radjai@umontpellier.fr ratios $I^{2}=\sigma_{i} / \sigma_{p}$ and cohesion index $\xi=\sigma_{c} / \sigma_{p}$. The dimensionless number $I$ is the inertial number of the dry granular flow, defined as the ratio of two time scales [relaxation time $\langle d\rangle\left(\rho_{s} / \sigma_{p}\right)^{1 / 2}$ under load versus shear time $\left.\dot{\gamma}^{-1}\right][23,24]$. Its relevance was demonstrated by showing that it controls the effective friction coefficient and packing fraction in different flow geometries [25-27]. The cohesion index $\xi$ quantifies the effective cohesion of the agglomerate in the presence of confining pressure, and it has been successfully used to scale cohesive aggregates and flows [28-30].

The behavior of the agglomerate is not, however, restricted to the two extreme limits of survival and disintegration. The primary particles located at the surface of an agglomerate can be eroded by frictional and inertial forces exerted by the surrounding granular flow. The attrition reflects the inhomogeneous distribution of contact forces inside the granular flow, and can lead to progressive loss of peripheral particles even in a strong agglomerate [19,31]. The agglomerate can also lose its cohesive bonds and break up into smaller agglomerates or simply elongate with or without loss of primary particles or cohesive bonds as a result of the action of shear stresses exerted by the flow. These failure modes and transitions between them are controlled by $I$ and $\xi$.

In cohesive granular flows, particle dynamics simulations in two dimensions (2D) show that a combination of $I$ and $\xi$ can be used to scale the rheological properties [28]. In the same way, in dense suspensions where both viscous and inertial forces come into play, the effective suspension viscosities are scaled by a combination of $I$ and the viscous number $\eta \dot{\gamma} / \sigma_{p}$, where $\eta$ is the liquid viscosity $[32,33]$. The case of agglomerates embedded in a dry flow is, however, a very different case since the cohesive forces are involved only in the interactions between wet primary particles inside the agglomerate, whereas inertial effects concern mainly dry 
particles in the surrounding fluid. For the granulation process, where the flow-agglomerate interactions play a crucial role, most investigations have focused on granulators where the packing fraction is low enough to consider only binary collisions between particles [11]. In dense granular flows, the issues of agglomerate nucleation, coalescence, and growth have been investigated, but it is generally found that the behavior is controlled by capillary, viscous, and frictional forces in a complex manner so that the relative importance of the forces can vary greatly with strain rate and liquid viscosity [10,34-41]. In a recent work, Lefebvre et al. investigated the erosion of a fixed wet agglomerate of particles subjected to a flow of dry particles inside a half-filled rotating drum [31]. They found that the erosion of the agglomerate is linear as a function of time and the force fluctuations play an important role for the erosion rate, but the erosion characteristic time is controlled by the liquid viscosity.

In this paper, we study the evolution of a single agglomerate introduced in a shear flow of dry particles by means of 3D numerical simulations. The simulations were performed by means of the discrete element method (DEM) in which the theoretical expressions of the capillary bridge force and lubrication viscous force as a function of interparticle distance are implemented in addition to the elastic and friction forces for their contact. We investigate the cumulative erosion (loss of particles from the agglomerate surface), breakage (loss of cohesive bonds), and elongation of the agglomerate for a broad range of values of the shear rate $\dot{\gamma}$ and surface tension $\gamma_{s}$ but keeping the same value of liquid viscosity. We use these data to analyze in detail the rates of these dispersion modes (erosion, breakage, and elongation). As we shall see, the scaling properties of these modes involve nontrivial combinations of the control parameters.

In Sec. II, we introduce the numerical procedures and parameters. We illustrate different dispersion modes of the agglomerate in Sec. III. The rates of erosion, breakage, and elongation, as well as their scalings with control parameters, are presented in Sec. IV. We conclude in Sec. V with a summary of remarkable results and future directions of research.

\section{MODEL DESCRIPTION AND PARAMETERS}

\section{A. Numerical method}

The discrete element method (DEM) [42-45] used in this work is a variant of the broad class of particle dynamics methods such as molecular dynamics (MD) for the simulation of molecular systems [46] and contact dynamics (CD) for the simulation of systems involving frictional contacts [47-49]. In the basic DEM, the particles interact via local force laws [50]. These forces are functions of the contact strains, which are defined from the relative displacements of the particles since no degrees of freedom other than rigid-body motions of the particles are considered. The particle displacements are calculated by stepwise resolution of Newton's second law:

$$
\begin{aligned}
m_{i} \frac{d^{2} \mathbf{r}_{i}}{d t^{2}} & =\sum_{j}\left[\left(f_{n}^{i j}+f_{c}^{i j}+f_{\mathrm{vis}}^{i j}\right) \mathbf{n}^{i j}+f_{t}^{i j} \mathbf{t}^{i j}\right], \\
\mathbf{I}_{i} \frac{d \boldsymbol{\omega}_{i}}{d t} & =\sum_{j} f_{t}^{i j} \mathbf{c}^{i j} \times \mathbf{t}^{i j}
\end{aligned}
$$

where particle $i$ is assumed to interact with its neighbors $j$ via normal contact forces $f_{n}$, tangential contact forces $f_{t}$, capillary forces $f_{c}$, and viscous forces $f_{\text {vis }}$. $\boldsymbol{\omega}_{i}$ is the rotation vector of the particle, and $m_{i}, \mathbf{I}_{i}$, and $\mathbf{r}_{i}$ are the mass, inertia matrix, and position of particle $i$, respectively. $\mathbf{n}^{i j}$ denotes the unit vector perpendicular to the contact plane between the particles $i$ and $j$ and pointing from $j$ to $i$. $\mathbf{t}^{i j}$ is the unit vector in the contact plane pointing in the direction opposite to the relative tangential displacement of the two particles. $\mathbf{c}^{i j}$ is the vector joining the center of particle $i$ to the contact point with particle $j$. We used a velocity-Verlet time-stepping scheme for the integration of the equations of motion [46].

The normal contact force $f_{n}$ is the sum of two contributions:

$$
f_{n}=f_{n}^{e}+f_{n}^{d}
$$

The elastic force $f_{n}^{e}=k_{n} \delta_{n}$ is a linear function of the normal elastic deflection $\delta_{n}$, where $k_{n}$ is the normal stiffness, and the damping force $f_{n}^{d}=\gamma_{n} \dot{\delta}_{n}$ is proportional to the relative normal velocity $\dot{\delta}_{n}$, where $\gamma_{n}$ is the normal viscous damping parameter. These forces occur only when two particles are in contact $\left(\delta_{n}<0\right)$. In the same way, the tangential force $f_{t}$ is composed of an elastic force $f_{t}^{e}=k_{t} \delta_{t}$ and a damping force $f_{t}^{d}=\gamma_{t} \dot{\delta}_{t}$, where $k_{t}$ is the tangential stiffness, $\gamma_{t}$ is the tangential damping parameter, and $\delta_{t}$ and $\dot{\delta}_{t}$ are the tangential displacement and velocity, respectively. According to the Coulomb friction law, the tangential force is below or equal to $\mu f_{n}$, where $\mu$ is the friction coefficient [50-52]:

$$
f_{t}=-\min \left\{\left|k_{t} \delta_{t}+\gamma_{t} \dot{\delta}_{t}\right|, \mu\left|f_{n}\right|\right\} \operatorname{sgn}\left(\dot{\delta}_{t}\right) .
$$

We assume that the liquid inside the agglomerate is in the "pendular" state with a homogeneous distribution of capillary bridges between particles [53-58]. This distribution may be a consequence of mixing the liquid with the particles, drainage of a saturated packing, or capillary condensation from a vapor. During the agglomeration process, the initial stage of nucleation and consolidation of an agglomerate involves the diffusion and redistribution of the liquid [59]. Once an agglomerate is formed, it can be assumed that the liquid is mainly carried by wet particles [15]. Consistently, the particles are assumed to be covered by the liquid so that the contact angle equals zero independently of liquid viscosity. For a separation distance above a debonding distance $d_{\text {rupt }}$, the bridge breaks and its liquid is shared between the two particles proportionally to their sizes [53,60]. As the agglomerate is embodied in a dry granular flow, we also assume that a wet particle leaving the agglomerate loses definitely its liquid by drainage, diffusion, or evaporation, and therefore it can no longer be captured by the agglomerate.

The capillary force $f_{c}$ between two particles depends on the liquid volume $V_{b}$ of the bond, liquid-vapor surface tension $\gamma_{s}$, and particle-liquid-gas contact angle $\theta$, as shown in Fig. 1(a). We used the following expression [61]:

$$
f_{c}= \begin{cases}-\kappa R & \text { for } \delta_{n}<0 \\ -\kappa R e^{-\delta_{n} / \lambda} & \text { for } 0 \leqslant \delta_{n} \leqslant d_{\text {rupt }} \\ 0 & \text { for } \delta_{n}>d_{\text {rupt }}\end{cases}
$$




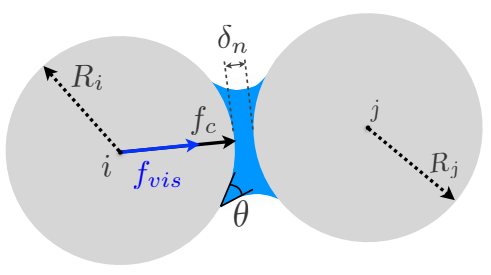

(a)

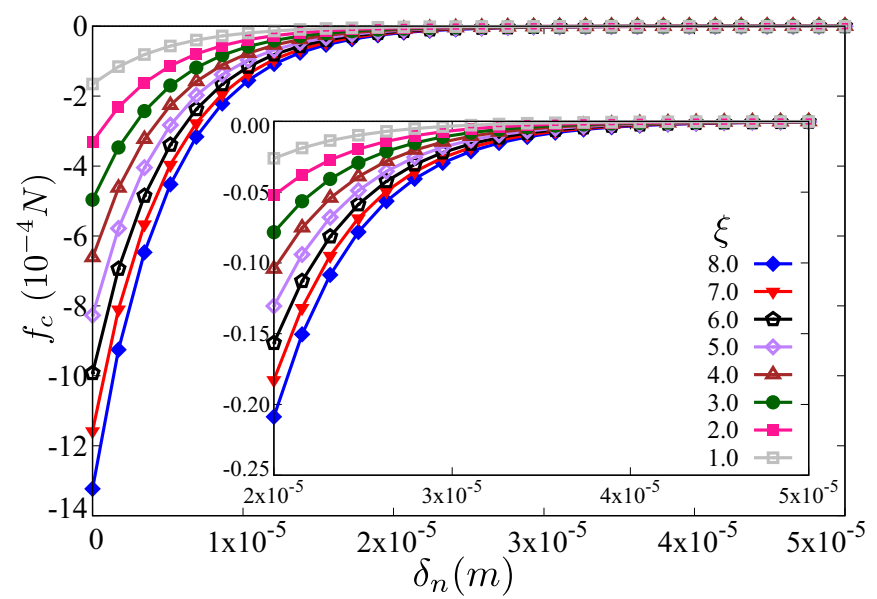

(b)

FIG. 1. (a) A capillary bridge between two wet particles $i$ and $j$, (b) capillary attraction force $f_{c}$ as a function of the separation distance $\delta_{n}$ up to the rupture distance $d_{\text {rupt }}$ for different values of the cohesion index $\xi$.

where $R=\sqrt{R_{i} R_{j}}$ is the geometrical mean radius, and the prefactor $\kappa$ is

$$
\kappa=2 \pi \gamma_{s} \cos \theta .
$$

This expression is an approximate solution of the YoungLaplace equation, and it was found to provide an excellent agreement with experimental data on the cohesion of wet granular materials [61]. The debonding distance $d_{\text {rupt }}$ is given by [53]

$$
d_{\text {rupt }}=\left(1+\frac{\theta}{2}\right) V_{b}^{1 / 3} .
$$

The characteristic length $\lambda$ in Eq. (4) is given by

$$
\lambda=\operatorname{ch}(r)\left(\frac{V_{b}}{R^{\prime}}\right)^{1 / 2},
$$

where $R^{\prime}=2 R_{i} R_{j} /\left(R_{i}+R_{j}\right)$ and $r=\max \left\{R_{i} / R_{j} ; R_{j} / R_{i}\right\}$ are the harmonic mean radius and the size ratio between two particles, $h(r)=r^{-1 / 2}$, and $c \simeq 0.9$ [55]. Figure 1(b) displays the capillary force as a function of the gap between two particles for different values of surface tension.

The normal lubrication force $f_{\text {vis }}$ due to the extension or contraction of the liquid bridge between two smooth spherical particles is given by $[31,62]$

$$
f_{\text {vis }}=\frac{3}{2} \pi R^{2} \eta \frac{v_{n}}{\delta_{n}},
$$

where $\eta$ is the liquid viscosity and $v_{n}$ is the relative normal velocity, assumed to be positive when the gap $\delta_{n}$ is decreasing.

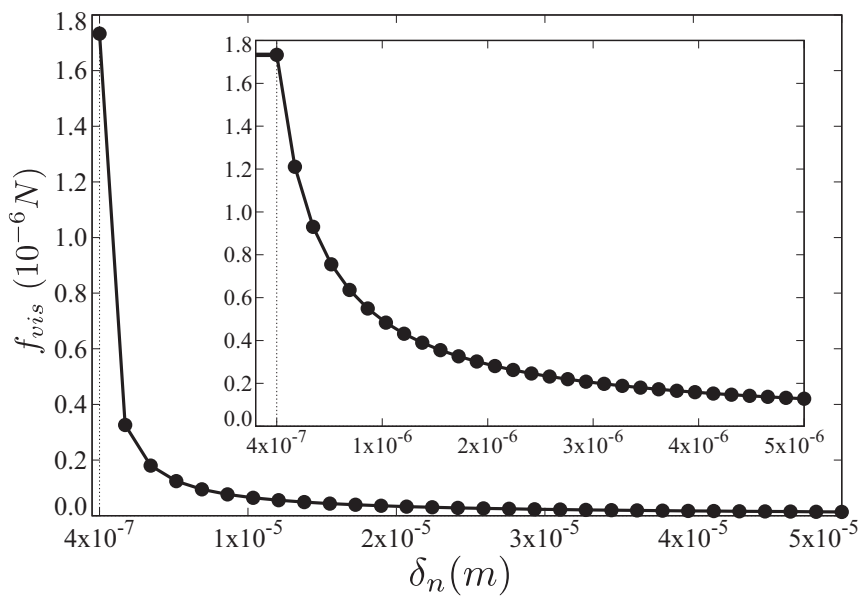

FIG. 2. Viscous force $f_{\text {vis }}$ as a function of the gap $\delta_{n}$ between two particles for given values of the relative normal velocity $\left(5 \times 10^{-3}\right.$ $\mathrm{m} / \mathrm{s})$ and liquid viscosity $(\eta=1 \mathrm{mPa} \mathrm{s})$.

This force diverges when the gap $\delta_{n}$ tends to zero. But for slightly rough particles, the characteristic size of the asperities allows for collision in finite time. Hence, we introduce a characteristic length $\delta_{n 0}$ corresponding to the size of asperities so that the lubrication force for $\delta_{n}>0$ is given by

$$
f_{\mathrm{vis}}=\frac{3}{2} \pi R^{2} \eta \frac{v_{n}}{\delta_{n}+\delta_{n 0}} \text { for } \delta_{n}>0 .
$$

For $\delta_{n}<0$ (a contact between two particles), we assume that the lubrication force remains equal to its largest value:

$$
f_{\text {vis }}=\frac{3}{2} \pi R^{2} \eta \frac{v_{n}}{\delta_{n 0}} \quad \text { for } \delta_{n} \leqslant 0 .
$$

Figure 2 shows $f_{\text {vis }}$ as a function of $\delta_{n}$ between two particles. In our simulations, we set $\delta_{n 0}=5 \times 10^{-4} d_{\min }$, where $d_{\min }$ is the smallest particle diameter. This value is sufficiently small to allow the lubrication force to be effective without leading to its divergence at contact.

\section{B. Samples}

We first prepared a dense sample of $N_{p}=20000$ spherical particles by means of isotropic compaction inside a rectangular box under the action of a compressive stress $\sigma_{p}$. We introduced a small size polydispersity by distributing the particle diameters randomly in the range $\left[d_{\min }, d_{\max }=2 d_{\min }\right]$. The top and bottom walls were made rough by gluing an array of spherical particles of diameter $d_{w}=2.23 d_{\min }$ to them. In the second step, we removed the lateral walls along the $x$ and $y$ directions and replaced them by periodic boundary conditions. A spherical probe was introduced in the granular bed and its diameter was increased until reaching exactly 300 particles inside the probe; see Fig. 4(a). Then, the capillary attraction forces and viscous forces were activated between neighboring particles inside the probe, creating thus a wet agglomerate of 300 particles inside a bed of dry particles. In the steady flow state, the height $h$ of the simulation box is $\sim 35$ mean particle diameters. The size of the agglomerate was chosen based on the size of the simulation box (the particle size needs to be small compared to the box size in order to avoid wall effects) 
TABLE I. Simulation parameters.

\begin{tabular}{lccc}
\hline \hline Parameter & Symbol & Value & Unit \\
\hline Smallest particle diameter & $d_{\min }$ & 800 & $\mu \mathrm{m}$ \\
Density of particles & $\rho_{s}$ & 2600 & $\mathrm{~kg} \mathrm{~m}^{-3}$ \\
Number of particles & $N_{p}$ & 20000 & \\
Friction coefficient & $\mu$ & 0.3 & \\
Normal stiffness & $k_{n}$ & $10^{6}$ & $\mathrm{~N} / \mathrm{m}$ \\
Tangential stiffness & $k_{t}$ & $8 \times 10^{5}$ & $\mathrm{~N} / \mathrm{m}$ \\
Normal damping & $\gamma_{n}$ & 0.8 & $\mathrm{Ns} / \mathrm{m}$ \\
Tangential damping & $\gamma_{t}$ & 0.8 & $\mathrm{Ns} / \mathrm{m}$ \\
Contact angle & $\theta$ & 0 & $\mathrm{deg}$ \\
Surface tension & $\gamma_{s}$ & {$[0.057,0.458]$} & $\mathrm{N} / \mathrm{m}$ \\
Liquid viscosity & $\eta$ & 1.0 & $\mathrm{mPa} \mathrm{s}$ \\
Time step & $\delta t$ & $7 \times 10^{-7}$ & $\mathrm{~s}$ \\
\hline \hline
\end{tabular}

and computation time (limited number of particles). Up to these limitations, the number of particles in the agglomerate should be high enough to allow for measurable evolution by elongation, erosion, or damage. Physically, the interactions between particles involve no length scale affecting the subsequent damage and deformations of the agglomerate. As we shall see, the behavior of the agglomerate basically depends on dimensionless parameters. Moreover, other studies of wet agglomerates under compression between two plates also suggest that the agglomerate size does not affect the compressive strength, which is basically set by the action of capillary forces [19].

To shear the sample, we applied a constant velocity $v$ along the $x$ direction to the top wall while keeping the bottom wall immobile and a constant pressure $\sigma_{p}$ on the top wall. We ran a total number of 64 simulations for different values of $v$ and $\gamma_{s}$. All other parameters are given in Table I. The samples were sheared for about $3 \mathrm{~s}$ in steady flow. Figures 3(a) and 3(b) show the mean velocity profiles for different values of the cohesion index $\xi$ and inertial number $I$, respectively. We see that, due to the choice of wall roughness, the shear deformation is nearly uniform despite the presence of the wet agglomerate inside the sample and independently of the values of $\xi$ and $I$. Hence, the steady flows can be characterized by an average shear rate $\dot{\gamma}=v / h$, where $h$ is the sample thickness, and an inertial number

$$
I=\dot{\gamma}\langle d\rangle\left(\frac{\rho_{s}}{\sigma_{p}}\right)^{1 / 2},
$$

where $\langle d\rangle$ denotes the mean particle diameter and $\rho_{s}$ is the particle density. We varied $\dot{\gamma}$ in the range $[0.21,39.68] \mathrm{s}^{-1}$. For the definition of the cohesion index, we used the maximum value of the capillary attraction force $f_{c}$ and confining force $f_{p}=\sigma_{p}\langle d\rangle^{2}$ :

$$
\xi=\frac{f_{c}}{f_{p}}=\frac{\kappa R}{\sigma_{p}\langle d\rangle^{2}} \approx \frac{\pi \gamma_{s} \cos \theta}{\sigma_{p}\langle d\rangle} .
$$

The cohesion index was varied in a range $[1,8]$.

\section{TIME EVOLUTION OF THE AGGLOMERATE}

Figure 4 displays snapshots of the agglomerate in different regimes depending on the values of $\xi$ and $I$. The agglomerate
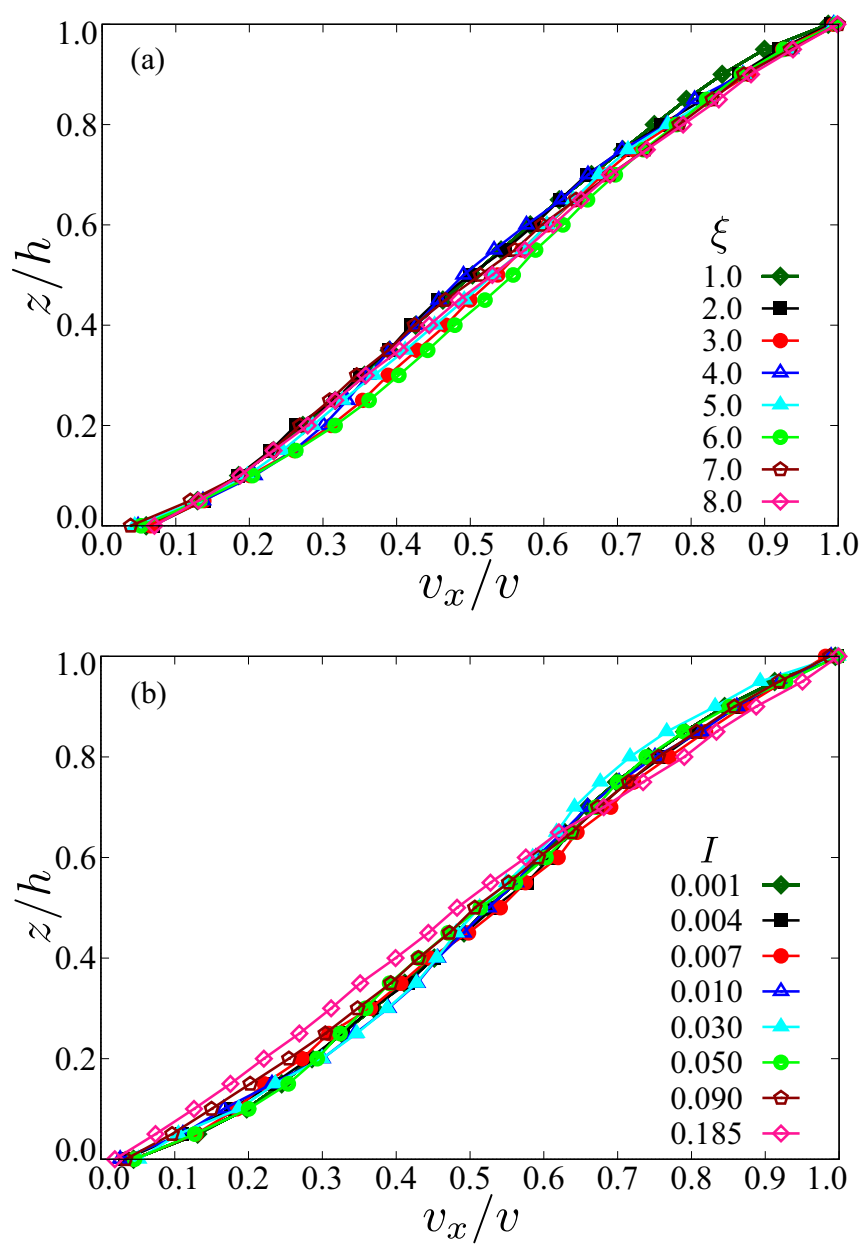

FIG. 3. Velocity profiles in steady shear state for different values of the cohesion index $\xi$ (a) with $I \simeq 10^{-3}$, and for different values of the inertial number $I$ with $\xi=5.0$ (b).

is stable with no loss of wet particles or breakage at high values of $\xi$ and low values of $I$. But it undergoes either sudden dispersion (disintegration) or gradual dispersion by loss of wet particles (erosion), loss of cohesive bonds (breakage), or elongation in other cases. These last three modes of dispersion occur simultaneously. Spontaneous dispersion occurs at low values of $\xi$, in which case the agglomerate behaves as the surrounding cohesionless particles and disappears as its primary particles follow shear flow.

The erosion, breakage, and elongation modes occur as a result of interactions between wet primary particles at the surface of the agglomerate and surrounding dry particles. We observed no breakage of the agglomerate into several smaller agglomerates or large lumps of wet particles. Hence, the evolution of the agglomerate can simply be measured by considering the cumulative number of primary particles leaving the initial agglomerate and the deformation of the remaining particles. We define the cumulate elongation $M_{g}$ of the agglomerate as the logarithm of the length of its long axis $\ell$ divided by its mean diameter $D$ :

$$
M_{g}=\ln \frac{\ell}{D}
$$




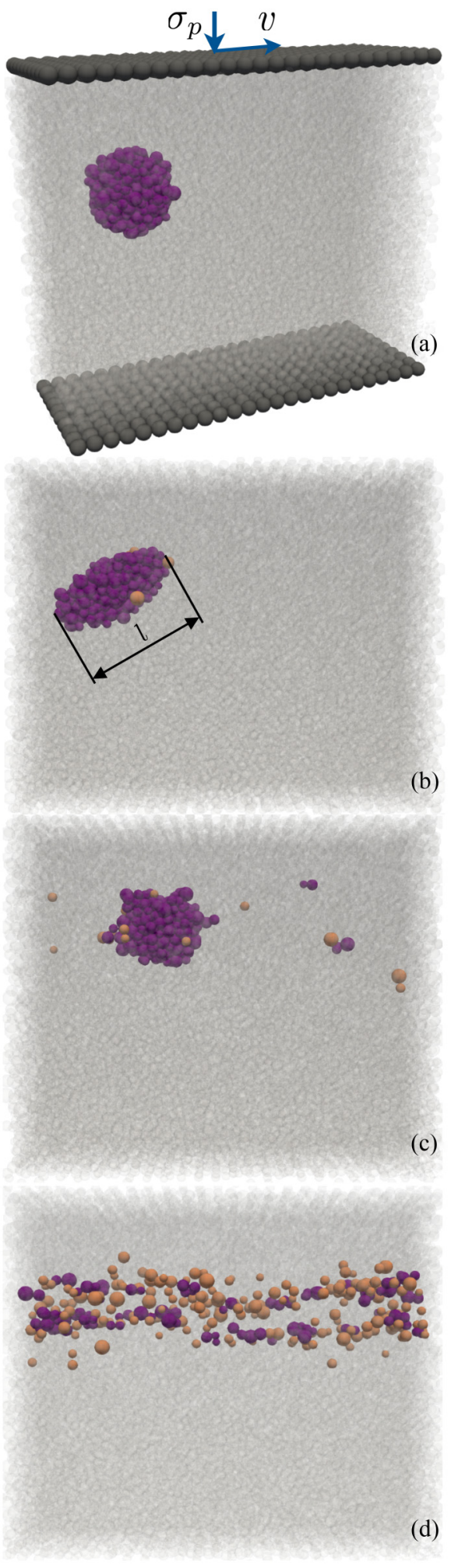

FIG. 4. (a) Simulated model of a wet agglomerate in a granular bed of dry particles subjected to a homogeneous shear flow; (b) elongation and erosion of the agglomerate; (c) elongation, erosion, and breakage modes; (d) spontaneous dispersion of wet particles.

The logarithm function arises as a result of time integration of incremental deformation $\delta \ell / \ell$ from the initial size $D_{0}$ to the current size $\ell$. Note that incremental deformation fluctuates in time due to the low number of particle diameters in the
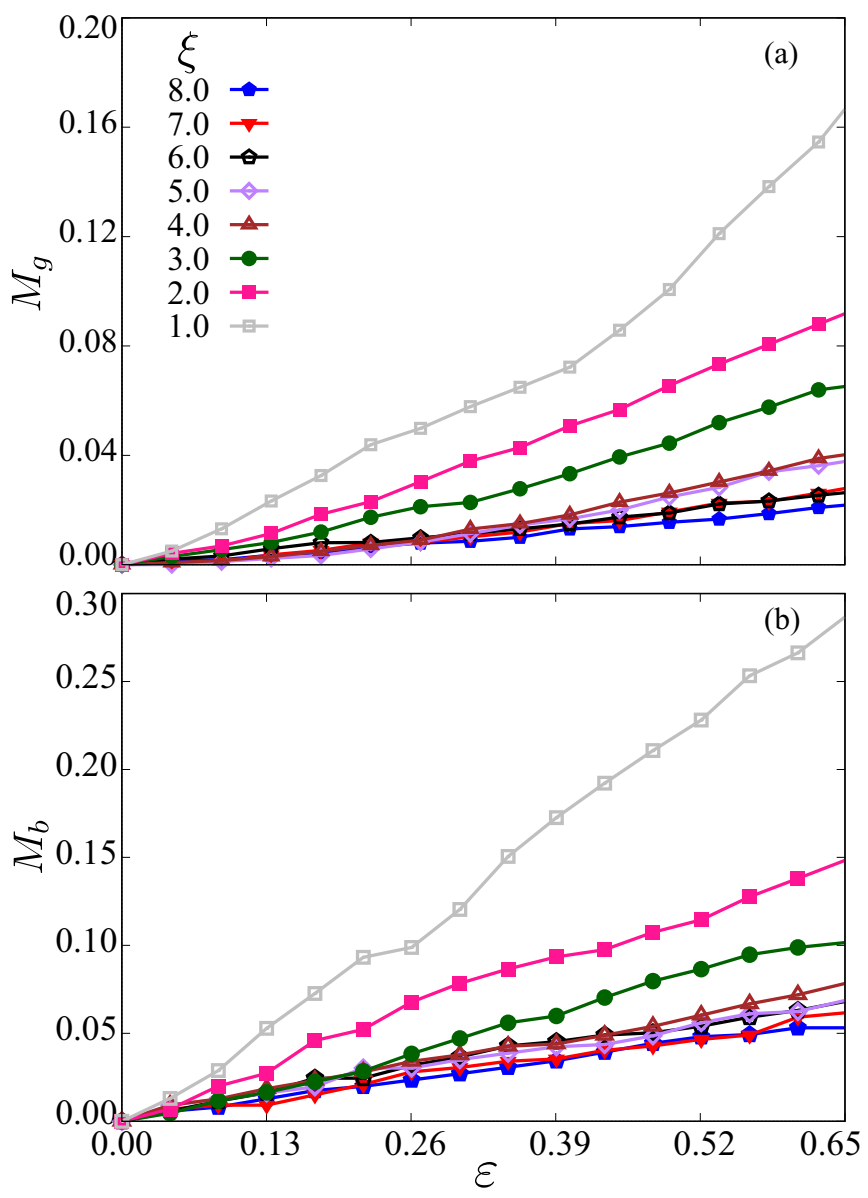

FIG. 5. Evolution of cumulative elongation $M_{g}$ (a) and breakage $M_{b}$ (b) of a wet agglomerate inside a homogeneous shear flow of dry particles as a function of cumulative shear strain $\varepsilon$ for different values of the cohesion index $\xi$ and for $I \simeq 10^{-3}$.

agglomerate. But the fluctuations average out by cumulating the increments over increasingly longer time.

The cumulative breakage $M_{b}$ of the agglomerate is the ratio of the number Nlost $t_{c}$ of broken capillary contacts to the total number of capillary contacts $N_{c i}$ at the initial state:

$$
M_{b}=\frac{N l o s t_{c}}{N_{c i}} .
$$

In the same way, the cumulative erosion $M_{e}$ is the ratio of the number Nlost ${ }_{p}$ of wet particles leaving the agglomerate to the total initial number $N_{p i}$ of wet particles:

$$
M_{e}=\frac{\text { Nlost }_{p}}{N_{p i}} .
$$

Note that erosion always implies the breakage and loss of contacts. But breakage can also take place inside the agglomerate without erosion. Elongation can be a consequence of the relative motions of the primary particles inside the agglomerate without erosion or a consequence of erosion.

Figure 5 shows $M_{g}$ and $M_{b}$ as a function of cumulative shear strain $\varepsilon=\dot{\gamma} t$ for $I \simeq 10^{-3}$ and all values of the cohesion index $\xi$. We see that both elongation and breakage increase as a nearly linear function of $\varepsilon$ at a rate that declines with increasing $\xi$. For the lowest values of the latter, the evolution 


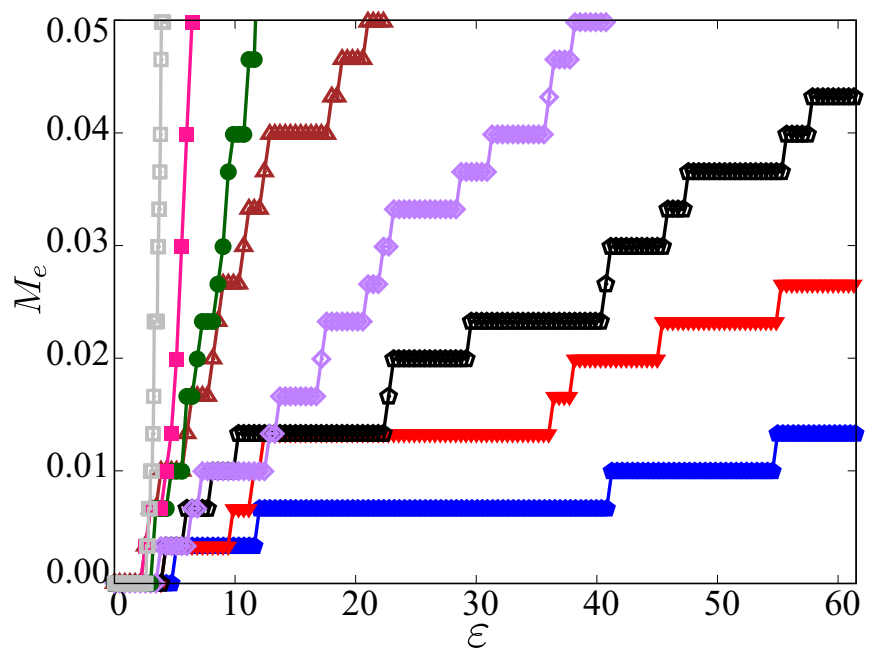

FIG. 6. Evolution of cumulative erosion as a function of shear strain $\varepsilon$ for different values of the cohesion index $\xi$ and for $I \simeq 0.1$.

is fluctuating with sometimes sudden changes of the rate. It is remarkable that both elongation and breakage occur even for the largest values of the cohesion index used in this work. We also observe a similarity between the evolutions of elongation and breakage. This reflects the fact that elongation implies the loss of capillary bonds inside the agglomerate as a result of the rearrangements of primary particles. The examples shown in Fig. 5 concern a low value of $I$. For larger values of $I$ the behavior is similar although the rates increase, as we shall see below.

Figure 6 shows the cumulative erosion $M_{e}$ as a function of shear strain for different values of $\xi$ for $I \simeq 0.1$. We observe a quick increase of $M_{e}$ at low values of $\xi$, corresponding to a quick dispersion of the wet particles. The erosion declines for larger values of cohesion index $\xi$. The number of eroded wet particles is small as they occur only occasionally. In fact, to increase the erosion rate, $\xi$ should be reduced, resulting in more breakage than erosion. Despite the stepwise evolution of erosion, it may be approximated by an average constant rate. This feature does not depend on $I$.

To characterize the three dispersion modes (elongation, breakage, and erosion) of the agglomerate, we consider their rates $K_{g}, K_{b}$, and $K_{e}$ evaluated from linear fits to the temporal evolutions of $M_{g}, M_{b}$, and $M_{e}$, respectively:

$$
\begin{aligned}
K_{g} & =\frac{\Delta M_{g}}{\Delta t}, \\
K_{b} & =\frac{\Delta M_{b}}{\Delta t} . \\
K_{e} & =\frac{\Delta M_{e}}{\Delta t},
\end{aligned}
$$

Figures 7(a), 7(b) and 7(c) display $K_{g}, K_{e}$, and $K_{b}$ as a function of the inertial number $I$ for different values of the cohesion index $\xi$. The symbols and their colors represent the same values of the cohesion index $\xi$ in the three figures. All the rates increase with increasing $I$ and decreasing $\xi$. While $K_{g}$ increases almost linearly with $I$ for all values of $\xi$, both $K_{e}$ and $K_{b}$ increase appreciably and in a nonlinear manner with $I$. Note also that, in contrast to breakage and elongation, for
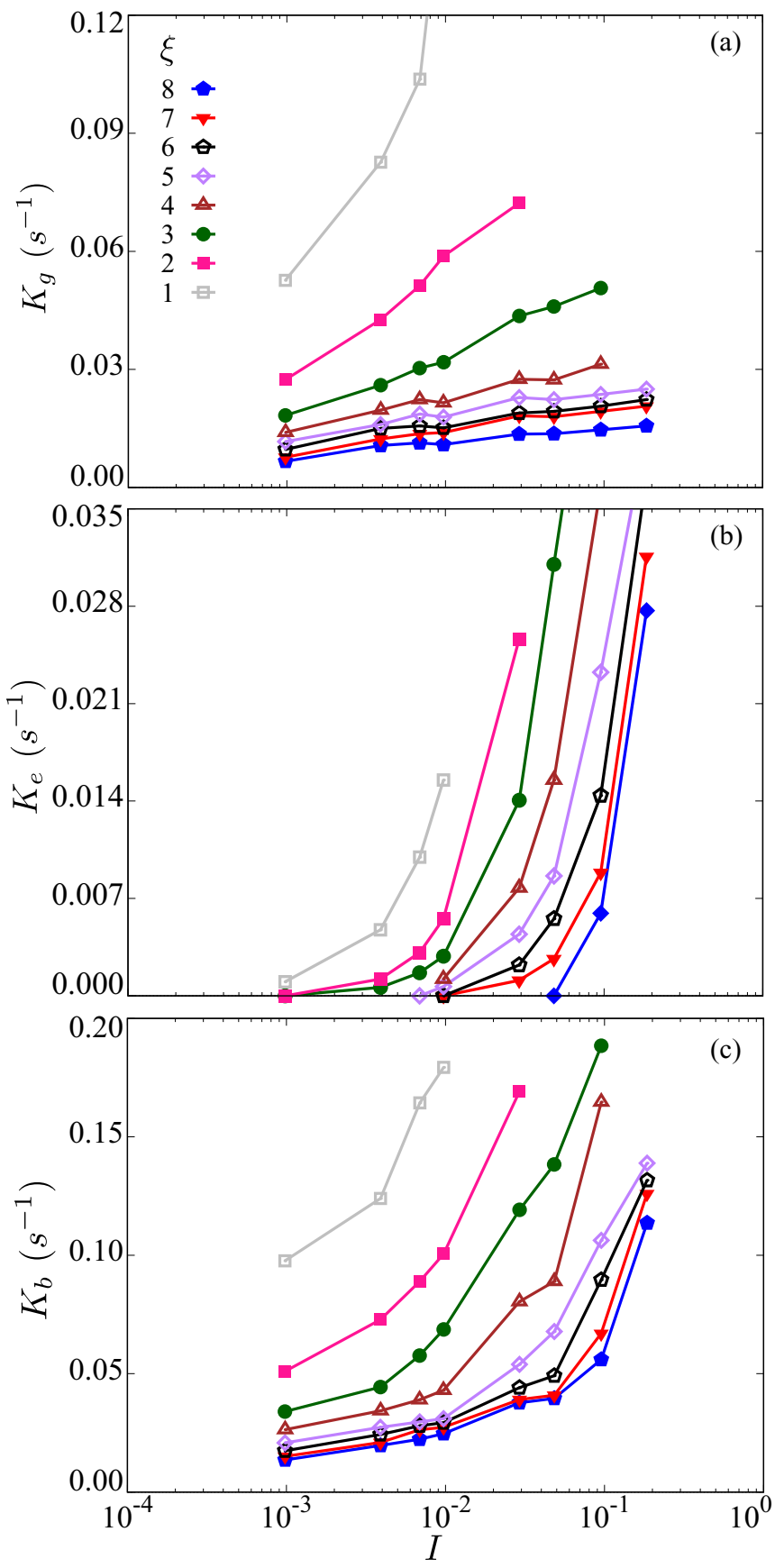

FIG. 7. Elongation rate $K_{g}$ (a), erosion rate $K_{e}(\mathrm{~b})$, and breakage rate $K_{b}$ (c) of the wet agglomerate as a function of the inertial number $I$ for different values of the cohesion index $\xi$.

each value of $\xi$ there is a lower threshold of $I$ below which no erosion occurs. This threshold increases with $\xi$.

The strong effect of increasing inertial number on the dispersion rates for all values of cohesion indicates that the inertial stresses prevail as compared to the confining pressure. Figure 8 shows the average normal force $\left\langle f_{n}\right\rangle$ and the average tangential force $\left\langle f_{t}\right\rangle$ between dry particles of the flow and wet particles located at the surface of the agglomerate as a function of $I$ for different values of $\xi$. These forces are responsible for both erosion of the particles from the agglomerate and its breakage. We see that, even for low values 

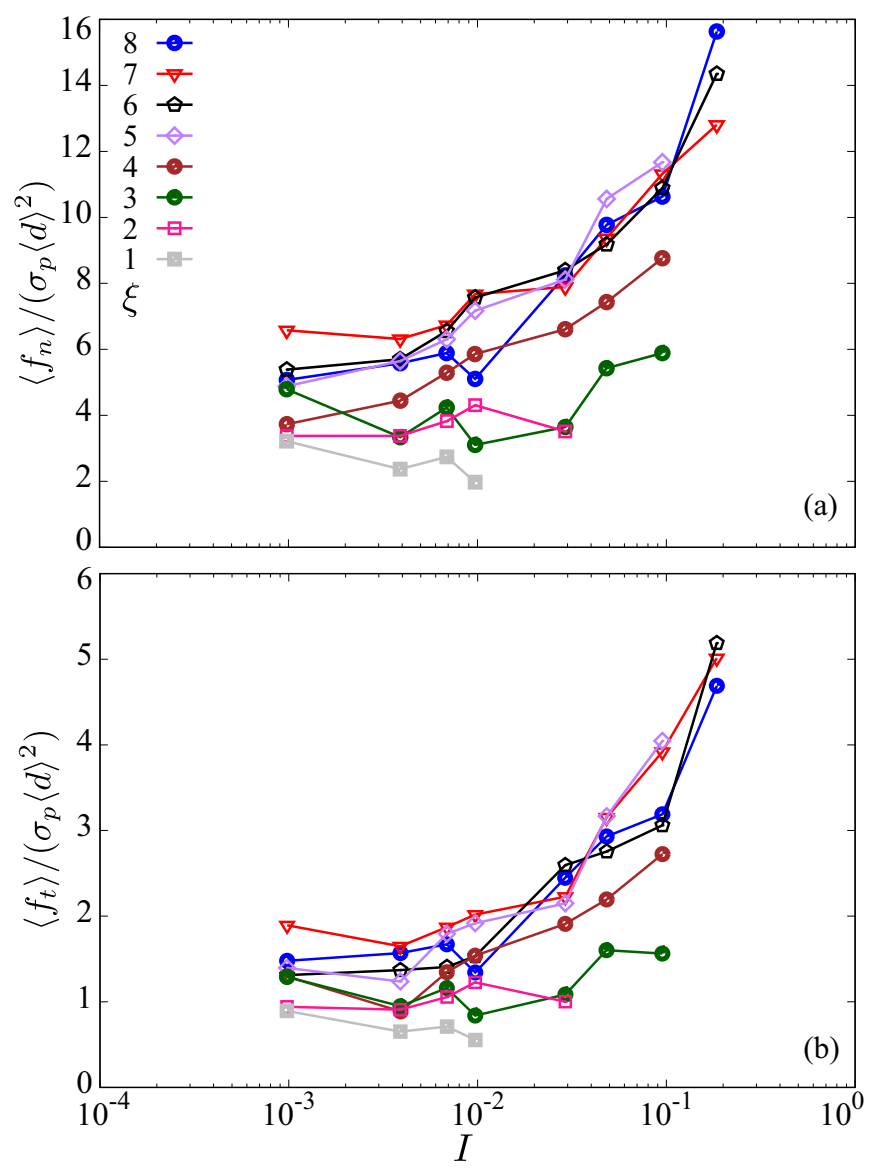

FIG. 8. Average normal force (a) and tangential force (b) at the contacts between the dry particles of the flow and the wet particles of the agglomerate as a function of the inertial number for different values of the cohesion index. The forces are normalized by the confining force $\sigma_{p}\langle d\rangle^{2}$.

of $I$, these forces are larger than the mean force exerted by the confining pressure on the particles. They increase rapidly as $I$ varies from $10^{-3}$ to $10^{-1}$. It is interesting to see that the average force increases for larger values of the cohesion index $\xi$. This can be a consequence of the fact that, at large cohesion, the dry particles "see" the agglomerate as a large rigid particle so that the momenta of the impacting particles are fully transmitted to the agglomerate. At low cohesion, the impacts may lead to erosion or breakage of the particle, resulting in the partial transmission of the impacting particle momenta to other particles.

\section{SCALING BEHAVIOR}

The behavior of the three dispersion rates in Fig. 7 as a function of $I$ and $\xi$ suggests that they may be separately collapsed on a master curve as a function of a scaling parameter combining $I$ and $\xi$. Before considering this option, it is necessary to define dimensionless rates by means of a characteristic time. There are three characteristic times in our system: (i) The relaxation time $\langle d\rangle \sqrt{\rho_{s} / \sigma_{p}}$ of the particles under the action of the confining pressure $\sigma_{p}$, (ii) the viscous time $\eta / \sigma_{p}$ due to viscous force, and (iii) the shear (inertial) time $t_{i}=\dot{\gamma}^{-1}$. The relaxation time is constant as the confining

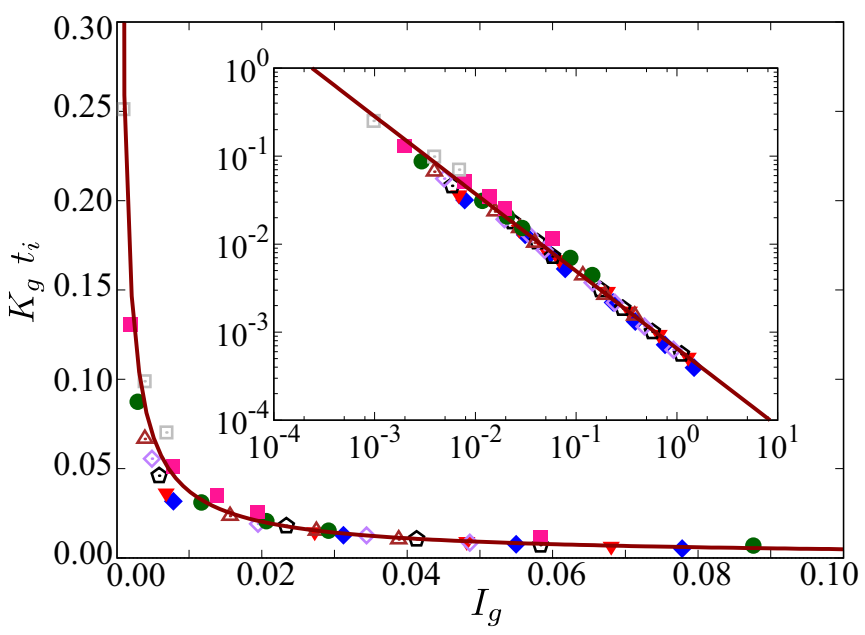

FIG. 9. Normalized elongation rate $K_{g} t_{i}$ as a function of the scaling parameter $I_{g}=\xi I^{\alpha_{g}}$ with $\alpha_{g}=1$ for all our simulations. The symbols and their colors represent the same values of the cohesion index $\xi$ as in Fig. 5. The solid line is a power-law fitting form [Eq. (20)].

stress has a fixed value $(=140 \mathrm{~Pa})$ in all simulations. Given the size of the particles, its value is small $\left(\sim 10^{-3} \mathrm{~s}\right)$. The viscous time has also a constant value in all simulations $\left(\sim 10^{-5} \mathrm{~s}\right)$. Hence, at the scale of the relaxation and viscous times, the dispersion events are rare and slow. In contrast, the shear time $t_{i}$ represents a time during which the agglomerate undergoes a long enough evolution for the erosion, elongation, and breakage events to be appreciable, although its value depends on the shear rate. Hence, we normalize all the times by the shear time $t_{i}$ so that the dimensionless shear rates are given by $K_{g} t_{i}, K_{b} t_{i}$, and $K_{e} t_{i}$.

We assume a general scaling parameter $I^{\alpha} \xi^{\beta}$ and search for the values of the exponents $\alpha$ and $\beta$ that make all the data points collapse on a single curve for each dispersion mode. Obviously, if the data points collapse for some values of these exponents, they will also collapse for any power of $I^{\alpha} \xi^{\beta}$, meaning that only the ratio $\alpha / \beta$ is relevant. Hence, we set the value of $\beta$ and determine $\alpha$ for each dispersion mode denoted by a subscript ( $g$ for elongation, $b$ for breakage, and $e$ for erosion).

Figures 9, 10, and 11 show the collapsed data on a master curve in linear and log-log scales for an appropriate choice of the exponents. For elongation and breakage, we set $\beta_{g}=$ $\beta_{b}=1$. Remarkably, within our statistical precision, we get $\alpha_{g}=\alpha_{b} \simeq 1$ with a good approximation. This means that the scaling parameter for elongation and breakage is

$$
I_{g}=I_{b} \simeq \xi I,
$$

with power-law functional fits

$$
K_{g} t_{i}=A_{g} I_{g}^{n_{g}},
$$

where $A_{g} \simeq 6.5 \times 10^{-4}$ and $n_{g} \simeq-0.88$, and

$$
K_{b} t_{i}=A_{b} I_{b}^{n_{b}}
$$

with $A_{b} \simeq 3.0 \times 10^{-3}$ and $n_{b} \simeq-0.65$.

This power-law scaling implies that the breakage and elongation of agglomerates occur for all values of $\xi$ and $I$, and no 


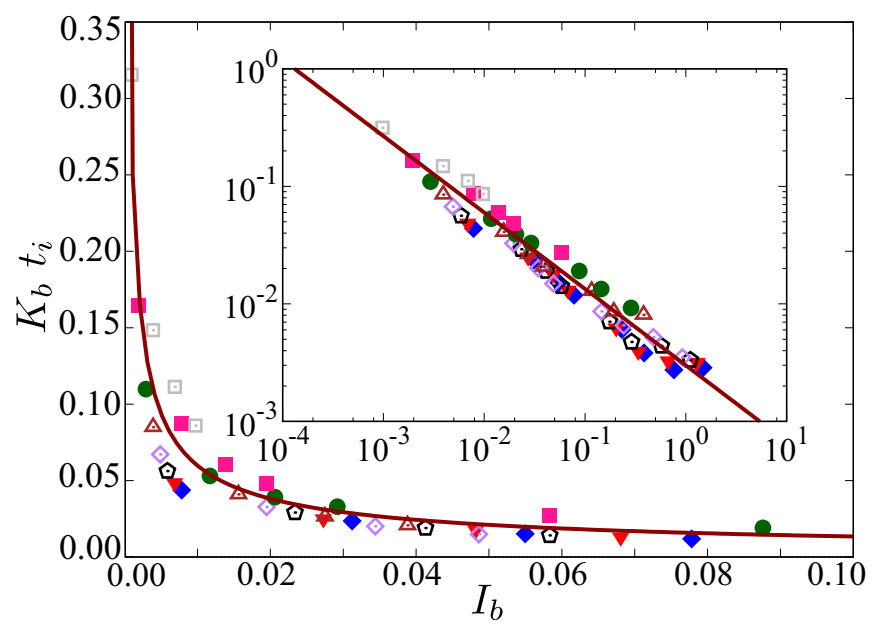

FIG. 10. Normalized breakage rate $K_{b} t_{i}$ as a function of the scaling parameter $I_{b}=\xi I^{\alpha_{b}}$ with $\alpha_{b}=1$ for all our simulations. The symbols and their colors represent the same values of the cohesion index $\xi$ as in Fig. 5. The solid line is a power-law fitting form [Eq. (21)].

transition occurs to a stable agglomerate state (no breakage, no elongation). If such a limit exists, it is out of the range of values of $I$ and $\xi$ investigated in this work. The negative values of the exponents $n_{g}$ and $n_{b}$ mean that the rates decrease both when $\xi$ increases (which is an expected behavior) and when $I$ increases, which is rather counterintuitive as inertial effects should enhance breakage. Actually, this is only a consequence of scaling the time by $t_{i}$. The temporal rates of breakage and elongation do increase with $I$ as observed in Fig. 7.

For erosion, we set $\beta_{e}=-1$, and the data points collapse for $\alpha_{e} \simeq 0.25$. Hence, the scaling parameter is

$$
I_{e}=I^{\alpha_{e}} \xi^{\beta_{e}} \simeq I^{1 / 4} / \xi
$$

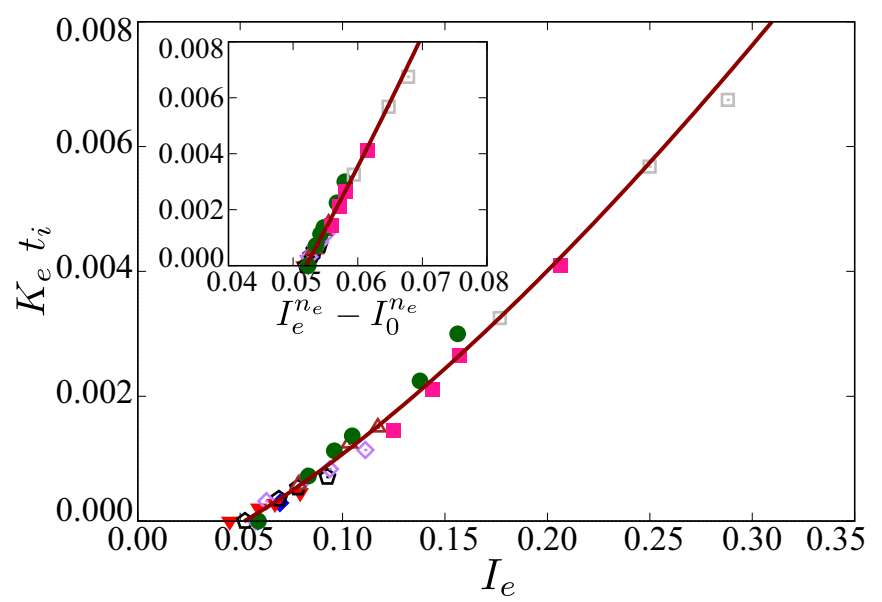

FIG. 11. Normalized erosion rate $K_{e} t_{i}$ as a function of the scaling parameter $I_{e} \simeq I^{\alpha_{e}} \xi^{\beta_{e}}$ with $\alpha_{e}=0.25$ and $\beta_{e}=-1$ for all our simulations. The symbols and their colors represent the same values of the cohesion index $\xi$ as in Fig. 5. The solid line is a truncated power-law fitting form [Eq. (23)].

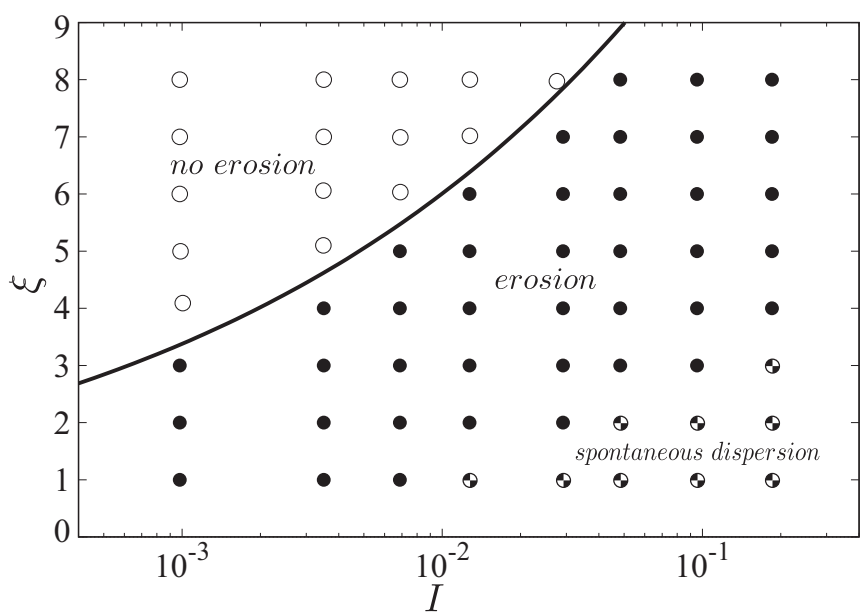

FIG. 12. Diagram of erosion states in the phase space $(I, \xi)$. The filled and empty symbols represent the states of erosion and vanishing erosion, respectively, for all the simulated values of $I$ and $\xi$ with a power-law frontier $\xi=I^{1 / 4} / I_{0}$ between them. The half-filled symbols correspond to the simulations where the agglomerate breaks up spontaneously, i.e., over one time step.

As displayed in Fig. 11, when plotted as a function of $I_{e}$, the data points collapse on a truncated power-law function:

$$
K_{e} t_{i}=A_{e}\left(I_{e}^{n_{e}}-I_{0}^{n_{e}}\right),
$$

with $A_{e} \simeq 4.5 \times 10^{-2}, I_{0} \simeq 5.2 \times 10^{-2}$, and $n_{e} \simeq 1.4$. The threshold $I_{0}$ is the value of $I_{e}$ below which no erosion occurs. Hence, in the phase space $(I, \xi)$, the function $\xi=I^{\alpha_{e}} / I_{0}$ defines the borderline between the states of erosion (below the curve) and vanishing erosion (above the curve), as shown in the phase diagram of Fig. 12.

The dispersion modes described and scaled here are not independent phenomena. For example, the elongation and erosion of the agglomerate imply gradual loss of contacts, which we count as part of breakage or damage of the agglomerate. As we can see in Fig. 7, for each value of the cohesion index, the three rates increase correlatively as a function of the inertial number. However, the dominant mode depends on the values of the cohesion index and inertial number. For example, from Eqs. (23) and (21), it is easy to see that, for each value of $\xi$, the dominant mechanism at low $I$ is erosion compared to breakage. As the inertial number increases, the ratio of erosion rate to breakage rate declines. The data presented in this paper do not include those simulations that led to a spontaneous dispersion of the agglomerate. In our analysis, this scenario, which happens only for low values of $\xi$ and the highest values of $I$, corresponds to the breakage of the agglomerate over one time step, i.e., a large value of the breakage rate. These simulations are marked by a different symbol in the phase diagram of Fig. 12.

\section{CONCLUSION}

In this paper, we used particle dynamics simulations to investigate three dispersion mechanisms of a wet agglomerate in a homogeneous flow of dry particles: (i) breakage (loss of cohesive bonds), (ii) erosion (loss of primary particles), 
and (iii) elongation. The inclusion of liquid in the simulations was based on the addition of capillary bridges between neighboring particles up to a debonding distance, as well as the assumption that the eroded particles lose their liquid and cannot be recaptured by the agglomerate. We varied two parameters: (i) the agglomerate cohesion index $\xi$ by varying the surface tension $\gamma_{s}$ of the liquid, and (ii) the flow inertial number $I$ by changing the shear rate $\dot{\gamma}$. The three mechanisms are simultaneously active, but their relative roles change with $\xi$ and $I$. The rates of breakage, erosion, and elongation are well defined and they were used for a parametric study of the evolution of the wet agglomerate.

As expected, the three dispersion rates increase with increasing $I$ and decreasing $\xi$. The effect of $I$ on the breakage and erosion rates is quite remarkable. A transition is observed for a particular value of $\xi$ depending on $I$ from a state of vanishing erosion to a state of erosion. We showed that the rate of evolution for each mechanism can be described by a master function of a scaling parameter combining $I$ and $\xi$. By fitting the collapsed data, we determined this function, which is a power law for the normalized breakage and elongation rates, and a truncated power law for the normalized erosion rate. The truncation is a consequence of transition from a state of vanishing erosion to erosion for a particular value of the scaling parameter. In the phase space $(I, \xi)$ this transition is characterized by a power-law borderline.

To define dimensionless rates, we normalized the time by the inertial time $t_{i}=\dot{\gamma}^{-1}$. The experiments reported in [31] suggested that the liquid viscosity sets the time scale of erosion. However, in our simulations the relevant time scale is the inertial time since the viscous time and relaxation time are both quite small. What is more, the dispersion phenomena are governed by the competing effects of the cohesive stresses and inertial stresses. The scaling proposed in this paper can be further examined by performing more simulations with larger values of liquid viscosity and different values of the confining pressure. A transition to the viscous regime is expected to occur for large values of viscosity $[32,33]$, where the Stokes number will come into play in addition to the cohesion index and inertial number. The erosion rate should decrease with increasing viscosity due to lubrication forces acting between particles. More generally, since the lubrication force depends on the shear rate, the effect of liquid viscosity is expected to counterbalance the effect of inertial number on the elongation, erosion, and breakage modes.

The analysis presented in this paper was made possible by using simple boundary conditions leading to a uniform shear flows. A more detailed analysis is underway in order to characterize also the microscopic mechanisms of erosion, breakage, and elongation. This work provides a quantitative ground for a better understanding of the agglomeration process in more complex geometries such as rotating drums, where the growth of granules is controlled by the probabilities of capture and loss of primary particles inside an inertial flow. When the rate of particle capture is determined as a function of the cohesion index and inertial number, it can be combined with the erosion rule obtained in this paper in order to develop an analytical model for the kinetics of agglomeration.

\section{ACKNOWLEDGMENTS}

We gratefully acknowledge financial support by the Ministry of Education and Training in Vietnam, Campus France and Labex NUMEV (University of Montpellier).
[1] J. Nimmo, in Encyclopedia of Soils in the Environment, edited by D. Hillel (Elsevier, Oxford, 2005), pp. 28-35.

[2] J. Sarkar and D. Dubey, J. Non-Newton. Fluid Mech. 234, 236 (2016).

[3] B. J. Ennis, G. Tardos, and R. Pfeffer, Powder Technol. 65, 257 (1991).

[4] E. Rondet, M. Delalonde, T. Ruiz, and J. P. Desfoursb, Chem. Eng. J. 164, 376 (2010).

[5] A. Barkouti, E. Rondet, M. Delalonde, and T. Ruiz, J. Food Eng. 111, 234 (2012).

[6] A. Nosrati, J. Addai-Mensah, and D. J. Robinson, Hydrometallurgy 125-126, 90 (2012).

[7] S. H. Chien, G. Carmona, L. I. Prochnow, and E. R. Austin, J. Environ. Qual. 32, 1911 (2003).

[8] P. Suresh, I. Sreedhar, R. Vaidhiswaran, and A. Venugopal, Chem. Eng. J. 328, 785 (2017).

[9] I. Talu, G. I. Tardos, and M. I. Khan, Powder Technol. 110, 59 (2000).

[10] S. Iveson, J. Beathe, and N. Page, Powder Technol. 127, 149 (2002).

[11] K. Saleh and P. Guigon, Handbook of Powder Technology, Granulation (Elsevier, Amsterdam, 2007), Vol. 11, pp. 323375.
[12] M. Ghadiri, A. D. Salman, M. Hounslow, A. Hassanpour, and D. W. York, Chem. Eng. Res. Des. 89, 499 (2011).

[13] N. Rahmanian, M. Ghadiri, and X. Jia, Powder Technol. 206, 53 (2011), 9th International Symposium on Agglomeration and 4th International Granulation Workshop, 2009.

[14] M. A. Behjani, N. Rahmanian, N. F. bt Abdul Ghani, and A. Hassanpour, Adv. Powder Technol. 28, 2456 (2017).

[15] T. Trung Vo, S. Nezamabadi, P. Mutabaruka, J.-Y. Delenne, E. Izard, R. Pellenq, and F. Radjai, Eur. Phys. J. E 42, 127 (2019).

[16] A. Taboada, N. Estrada, and F. Radjandotiuml, Phys. Rev. Lett. 97, 098302 (2006).

[17] F. Radjaï and V. Richefeu, Philos. Trans. R. Soc., A 367, 5123 (2009).

[18] B. Saint-Cyr, F. Radjai, J.-Y. Delenne, and P. Sornay, Phys. Rev. E 87, 052207 (2013).

[19] Thanh-Trung Vo, P. Mutabaruka, S. Nezamabadi, J.-Y. Delenne, E. Izard, R. Pellenq, and F. Radjai, Mech. Res. Commun. 92, 1 (2018).

[20] F. Radjai, I. Preechawuttipong, and R. Peyroux, in Continuous and Discontinuous Modeling of Cohesive Frictional Materials, edited by P. Vermeer, S. Diebels, W. Ehlers, H. Herrmann, S. Luding, and E. Ramm (Springer-Verlag, Berlin, 2001), pp. 148159. 
[21] F. Radjai, V. Topin, V. Richefeu, C. Voivret, J.-Y. Delenne, E. Azéma, and M. S. El Youssoufi, in Mathematical Modeling and Physical Instances of Granular Flows, edited by J. D. Goddard, J. T. Jenkins, and P. Giovine (AIP, New York, 2010), pp. 240260.

[22] R. A. Bagnold, Proc. R. Soc. Lond. A 225, 49 (1954).

[23] F. da Cruz, S. Emam, M. Prochnow, J.-N. Roux, and F. Chevoir, Phys. Rev. E 72, 021309 (2005).

[24] P. Jop, Y. Forterre, and O. Pouliquen, Nature (London) 441, 727 (2006).

[25] GDR-MiDi, Eur. Phys. J. E 14, 341 (2004).

[26] O. Pouliquen, C. Cassar, P. Jop, Y. Forterre, and M. Nicolas, J. Stat. Mech.: Theor. Exp. (2006) 7020.

[27] Y. Forterre and O. Pouliquen, Annu. Rev. Fluid Mech. 40, 1 (2008).

[28] N. Berger, E. Azéma, J.-F. Douce, and F. Radjai, EPLEurophys. Lett. 112, 64004 (2015).

[29] S. Khamseh, J.-N. Roux, and F. Chevoir, Phys. Rev. E 92, 022201 (2015).

[30] M. Badetti, A. Fall, D. Hautemayou, F. Chevoir, P. Aimedieu, S. Rodts, and J.-N. Roux, J. Rheol. 62, 1175 (2018).

[31] G. Lefebvre and P. Jop, Phys. Rev. E 88, 032205 (2013).

[32] M. Trulsson, B. Andreotti, and P. Claudin, Phys. Rev. Lett. 109, 118305 (2012).

[33] L. Amarsid, J.-Y. Delenne, P. Mutabaruka, Y. Monerie, F. Perales, and F. Radjai, Phys. Rev. E 96, 012901 (2017).

[34] S. P. Rwei, I. Manas-Zloczower, and D. L. Feke, Polym. Eng. Sci. 31, 558 (1991).

[35] M. I. Khan and G. I. Tardos, J. Fluid Mech. 347, 347 (1997).

[36] G. I. Tardos, K. P. Hapgood, O. O. Ipadeola, and J. N. Michaels, Powder Technol. 140, 217 (2004), 1st International Workshop on Granulation (Granulation across the length scales: linking microscopic experiments and models to real process operation).

[37] A. Scurati, D. Feke, and I. Manas-Zloczower, Chem. Eng. Sci. 60, 6564 (2005).

[38] A. Hassanpour, S. Antony, and M. Ghadiri, Chem. Eng. Sci. 62, 935 (2006).

[39] A. Hassanpour, S. J. Antony, and M. Ghadiri, Adv. Powder Technol. 18, 803 (2007).

[40] N. D. Vassileva, D. van den Ende, F. Mugele, and J. Mellema, Langmuir 23, 2352 (2007).
[41] D. Liu, Z. Wang, X. Chen, and M. Liu, Powder Technol. 336, 102 (2018).

[42] P. A. Cundall and O. D. L. Strack, Géotechnique 29, 47 (1979).

[43] H. J. Herrmann and S. Luding, Continuum Mech. Thermodyn. 10, 189 (1998).

[44] C. Thornton, Powder Technol. 109, 179 (1999).

[45] F. Radjai and F. Dubois, Discrete-element Modeling of Granular Materials (Wiley-Iste, Hoboken, 2011).

[46] M. P. Allen and D. J. Tildesley, Computer Simulation of Liquids (Oxford University Press, Oxford, 1987).

[47] J.-J. Moreau, Eur. J. Mech. A 13, 93 (1994).

[48] F. Radjai and V. Richefeu, Mech. Mater. 41, 715 (2009).

[49] F. Radjai, C. R. Mécanique 346, 263 (2018).

[50] S. Luding, in Physics of Dry Granular Media, NATO ASI Series E350, edited by H. J. Herrmann, J.-P. Hovi, and S. Luding (Kluwer Academic, Dordrecht, 1998), p. 285.

[51] J. Schäfer, S. Dippel, and D. E. Wolf, J. Phys. I 6, 5 (1996).

[52] S. Dippel, G. G. Batrouni, and D. E. Wolf, Phys. Rev. E 56, 3645 (1997).

[53] G. Lian, C. Thornton, and M. Adams, J. Colloid Interface Sci. 161, 138 (1993).

[54] M. Scheel, R. Seemann, M. Brinkmann, M. D. Michiel, A. Sheppard, and S. Herminghaus, J. Phys.: Condens. Matter 20, 494236 (2008).

[55] V. Richefeu, M. S. El Youssoufi, and F. Radjai, Phys. Rev. E 73, 051304 (2006).

[56] J.-Y. Delenne, V. Richefeu, and F. Radjai, J. Fluid Mech. 762, R5 (2012).

[57] T. Trung Vo, P. Mutabaruka, J.-Y. Delenne, S. Nezamabadi, and F. Radjai, Eor. Phys. J. Web Conf. 140, 08021 (2017).

[58] V. D. Than, S. Khamseh, A. M. A. Tang, J.-M. Pereira, F. Chevoir, and J.-N. Roux, J. Eng. Mech. 143, C4016001 (2017).

[59] S. M. Iveson, J. D. Litster, K. Hapgood, and B. J. Ennis, Powder Technol. 117, 3 (2001).

[60] C. Willett, M. Adans, S. Johnson, and J. Seville, Langmuir 16, 9396 (2000).

[61] V. Richefeu, F. Radjai, and M. S. E. Youssoufi, Eur. Phys. J. E 21, 359 (2007).

[62] J. Happel and H. Brenner, Low Reynolds Number Hydrodynamics (Martinus Nijhoff, Dordrecht, Netherlands, 1983). 